Research Article

\title{
Calprotectin as a New Sensitive Marker of Neutrophilic Inflammation in Patients with Bronchiolitis Obliterans
}

\author{
S. P. Jerkic $\mathbb{D}^{1}{ }^{1}$ F. Michel, ${ }^{1}$ H. Donath, ${ }^{1}$ E. Herrmann $\mathbb{D}^{2}{ }^{2}$ R. Schubert, ${ }^{1}$ M. Rosewich, ${ }^{1}$ \\ and S. Zielen $\mathbb{D}^{1}$ \\ ${ }^{1}$ Department for Children and Adolescents, Division of Allergology, Pulmonology and Cystic Fibrosis, Goethe University, \\ Frankfurt, Germany \\ ${ }^{2}$ Institute of Biostatistics and Mathematical Modeling, Goethe University, Frankfurt, Germany
}

Correspondence should be addressed to S. Zielen; stefan.zielen@kgu.de

Received 4 January 2020; Revised 10 March 2020; Accepted 4 April 2020; Published 1 May 2020

Academic Editor: Paola Migliorini

Copyright ( 2020 S. P. Jerkic et al. This is an open access article distributed under the Creative Commons Attribution License, which permits unrestricted use, distribution, and reproduction in any medium, provided the original work is properly cited.

\begin{abstract}
Introduction. Bronchiolitis obliterans $(\mathrm{BO})$ is a chronic disease in which persistent inflammation leads to obstruction and obliteration of the small airways. The aim of this study was to evaluate the value of calprotectin as an inflammatory marker in induced sputum. Methods. Twenty-eight patients suffering from BO and 18 healthy controls were examined. Lung function was measured by spirometry, body plethysmography, and lung clearance index (LCI). The induced sputum was obtained, cell counts were performed, and cytokines were measured using cytometric bead array (CBA). Calprotectin was quantified in the sputum and serum samples using commercially available sandwich ELISA. Results. Spirometry parameters including forced vital capacity (FVC), forced expiratory volume in 1 second (FEV1), and maximum expiratory flow rate at 25\% vital capacity (MEF25) were significantly lower in $\mathrm{BO}$ patients than in healthy controls, whereas the reserve volume (RV), RV to total lung capacity ratio (RV/TLC), and LCI were significantly increased. In sputum, calprotectin levels, neutrophils, and IL-8 were significantly elevated. Calprotectin levels correlated strongly with IL-8 and other biomarkers, neutrophils FEV1 and MEF25. In serum, calprotectin was significantly diminished in BO patients compared to controls. Conclusion. Lung function is severely impaired in BO patients. Calprotectin is significantly elevated in the sputum of $\mathrm{BO}$ patients and reflects ongoing neutrophilic inflammation.
\end{abstract}

\section{Introduction}

Bronchiolitis obliterans (BO) is a rare, chronic form of obstructive lung disease. Most early descriptions of $\mathrm{BO}$ were limited to case reports with autopsy findings. Typically, BO begins with an injury to the bronchiolar epithelium followed by an inflammatory reaction that progresses to airway fibrosis and potential luminal obliteration $[1,2]$. The initial severe insult is thought to affect the lower airways and can be caused by either a pathogen, postinfectious BO (PIBO), lung transplantation (LT) or bone marrow transplantation (BMT), or $\mathrm{BO}$ syndrome (BOS) [3-5]. This failure of resolution of initial and ongoing inflammation is likely to be an important part of the disease process of BO. There is an influx of inflammatory and immune cells. In combination with the proliferation of granulation tissue in the small airways, this leads to obstruction of the bronchioli with air trapping and hyperinflation $[1,2,6]$.

$\mathrm{BO}$ is defined clinically as a small airway disease with persistent air flow obstruction and air trapping as determined by lung function measurements. In general, this airflow obstruction is nonresponsive to bronchodilators. Most studies use spirometry to measure lung function in $\mathrm{BO}$, but current guidelines highlight the need for new sensitive lung function indices to detect early small airway impairment. In the last decade, the lung clearance index (LCI) has repeatedly been shown to be superior to the forced expiratory volume in 1 second (FEV1) to monitor small airway disease in different lung diseases. The LCI is a feasible and sensitive tool to detect small airway impairment in chronic lung diseases in children and adults [7-10]. To the best of our knowledge, only a few studies have used this technique in patients with BO $[11,12]$. 
Previous investigations have shown that BO is predominantly a neutrophilic disease of the small bronchioles featuring elevated levels of proinflammatory cytokines [5]. Analysis of the sputum of $\mathrm{BO}$ patients reveals consistent neutrophil inflammation, while the levels of inflammatory cytokines such as IL-1 $\beta$, IL-6, and IL- 8 have greater variability [13]. As progression patterns have not yet been defined and due to the predominantly persistent neutrophilic inflammation in a changing inflammatory pattern, calprotectin, which is found extensively in the cytoplasm, might serve as a valuable surrogate biomarker for $\mathrm{BO}$.

Calprotectin is a $36 \mathrm{kDa}$ zinc-binding protein that is universally found in the body and in the cytoplasm of neutrophils and macrophages. Calprotectin is released from activated neutrophils, leading to high concentrations in various fluids, such as plasma, serum, spinal fluid, synovial fluid, urine, saliva, or stool [14]. Therefore, calprotectin is increased predominantly in inflammatory processes in relevant organs. As calprotectin is released locally, it may be a sensitive indicator of local inflammation [15]. Calprotectin is known as an excellent surrogate biomarker for the inflammatory status of the gut in inflammatory bowel disease. Calprotectin is measured in stool samples as there is an influx of inflammatory cells in the inflamed gut tissue in IBD, and it is known to be correlated with disease activity $[14,16]$.

This study investigates the value of calprotectin and cytokines in the sputum of BO patients. Additionally, this study explores the value of calprotectin as an inflammatory marker and its correlation with cytokine levels and LCI.

\section{Methods}

2.1. Patients and Controls. We prospectively studied twentyeight patients with $\mathrm{BO}$, including 21 patients with $\mathrm{PIBO}$ and 7 patients with BOS following human stem cell transplantation (HSCT). The median age of the patient cohort was 14.2 (6.2-27.3) years. The diagnosis BO was made by the following criteria: medical history of infectious insult or BMT, persistent crackles on clinical examination, lung function with an FEV $1<75 \%$ and reversibility of less than $12 \%$. In addition, most of the patients presented with typical radiological features at the time of diagnosis. Other causes of chronic respiratory symptoms, such as cystic fibrosis (CF), immunodeficiencies, aspiration syndrome, or congenital heart disease, were excluded in all patients. In addition, 18 healthy control subjects with a median age of 16.2 (7.6-25) years were examined. Written consent from patients or caregivers was required for every subject. The study followed the ethical principles of the Declaration of Helsinki, the regulatory requirements, and the code of Good Clinical Practice. The study was approved by the responsible ethic committee at the Frankfurt University and registered at clinicaltrials.gov (NCT02627833).

2.2. Lung Function Tests. The lung function tests and reversibility testing were evaluated using a body plethysmograph (VIASYS Healthcare GmbH, Hoechberg, Germany). The following parameters were recorded: forced vital capacity (FVC), FEV1, FEV1/VC, 75\% of the maximum expiratory flow (FEF 75\%), reserve volume (RV), and the ratio of RV to total lung capacity (RV/TLC). The LCI was measured by EasyOne Pro Lab (ndd Medical Technologies, Andover, Massachusetts, USA).

Lung function tests (spirometry and body plethysmography) and the LCI were evaluated in all patients at routine clinical visits. If the patients were receiving beta-agonists, they were advised to discontinue administration 8 hours prior to testing, and long-acting beta-agonists were to be withdrawn 24 hours before the study. FVC, FEV1, FEF $75 \%$, and flow-volume curves were evaluated according to the American Thoracic Society (ATS) guidelines. Lung volumes (TLC, forced residual capacity (FRC), and RV) were measured by plethysmography following international recommendations [17].

2.3. Airway Reversibility. Bronchodilator reversibility was tested after inhalation of $400 \mu \mathrm{g}$ of salbutamol by a metered dose inhaler (MDI) with a spacer. Reversibility was defined as yes/no using a $\geq 12 \%$ and $200 \mathrm{ml}$ post bronchodilator change.

2.4. Sputum Collection and Processing and Cell Analysis. The patients and control subjects first inhaled salbutamol followed by nebulization with hypertonic saline solution at increasing concentrations of $3 \%, 4 \%$, and $5 \%$ every 7 minutes, as recently described $[18,19]$. The sputum was processed within 1 hour of collection. The sputum was quantified, and sputum plugs were selected from the samples and placed in preweighted Eppendorf tubes. They were treated with $4 \mathrm{x}$ weight/volume $0.1 \%$ dithiothreitol (DTT), and the samples were processed for 15 minutes on ice before the subsequent addition of $2 \mathrm{x}$ the weight/volume of phosphatebuffered saline (PBS). Samples were filtered through a $48 \mu \mathrm{m}$ mesh and centrifuged for 10 minutes at $790 \times \mathrm{g}$. Supernatants were stored at $-80^{\circ} \mathrm{C}$ until further analysis. The pellet was resuspended in $300 \mu \mathrm{l}$ of $2 \%$ PBS/BS. Subsequently, the mix was set to 30,000 cells/ml for cytospin analysis, which was stained with May-Grunwald-Giemsa for differential cell counting $[18,19]$. In total, 400 cells per slide were counted, and the percentages of neutrophils, lymphocytes, eosinophils, and macrophages were expressed as proportions of the total cell count.

2.5. Cytometric Bead Array (CBA). The concentrations of three cytokines were determined in sputum samples using the $\mathrm{BD}^{\mathrm{TM}} \mathrm{CBA}$ Flex Set System (BD Bioscience-PharMingen, San Diego, CA, USA) to measure IL-6, IL-8, and IL- $1 \beta$ levels. Each $\mathrm{BD}^{\mathrm{TM}}$ CBA Flex Set contained a one-bead population with distinct fluorescence intensity and both the appropriate phycoerythrin (PE) detection reagent and the standard. The tests were performed according to the manufacturer's instructions. To analyse the cytokines, we added the same concentration of DTT $(0.025 \%)$ as in the sputum supernatant to the standard curve and enzyme immunoassay buffer as described [20]. The lower detection limits of the cytokines were $1.2 \mathrm{pg} / \mathrm{ml}, 1.6 \mathrm{pg} / \mathrm{ml}$, and $1.0 \mathrm{pg} / \mathrm{ml}$ for IL-8, IL-6, and IL- $1 \beta$, respectively. 
2.6. Statistics and Data Analysis. Data were analysed using GraphPad Prism (GraphPad Software Inc., La Jolla, CA, USA) and Microsoft Excel. Group differences between BO patients and control subjects were analysed using the Kruskal-Wallis test or Mann-Whitney $U$ test depending on the normality and homogeneity of variance assumptions. A probability of $p<0.05$ was considered significant.

\section{Results}

As shown in Table 1, we enrolled 28 patients (median age, 14.2; range, 6-27; male/female ratio, 11/7). We compared the patients' data with those of 18 healthy control subjects (median age, 16.2; range, $7-25$; male/female ratio $16 / 12$ ) (Table 1).

3.1. Lung Function Test Results. Patients with $\mathrm{BO}$ revealed significantly compromised lung function testing. The FVC, FEV1, and the maximum expiratory flow rate at $25 \%$ vital capacity (MEF25) were significantly decreased, while RV/TLC and LCI were significantly increased in BO patients (BO: 12.1, 7.3-20.5; controls: 7.1, 6.0-8.4, $p<0.001$ ) compared to controls (Table 1).

3.2. Airway Reversibility. No airway reversibility was detected in the majority of $\mathrm{BO}$ patients (Figure 1). Airway reversibility testing after administration of $400 \mu \mathrm{g}$ of salbutamol was positive in only one patient with an FEV1 increase of $>12 \%$ and $>200 \mathrm{ml}$ and in three patients with FEV1 increases of $>12 \%$ but $<200 \mathrm{ml}$.

3.3. Analysis of Inflammatory Cells and Cytokine Levels in Induced Sputum. As shown in Figure 2, a significantly higher percentage of neutrophils (BO patients: 79\%, 15-91\%; control subjects: $14 \%, 1-49 \%, p<0.001)$ was observed in all the $\mathrm{BO}$ patients, whereas the control subjects showed predominantly alveolar macrophages (BO patients: $20 \%, 5-83 \%$; control subjects: $86 \%, 48-99 \%, p<0.001)$ in their sputum samples.

All tested cytokines were detected in the sputum supernatants of BO patients and 19 control subjects. As shown in Figure 2, the levels of IL-1 $\beta$, IL-6, and IL- 8 were higher in the $\mathrm{BO}$ patients than in the controls, but only IL-8 levels (BO: $9855 \mathrm{pg} / \mathrm{ml}, 182-155228 \mathrm{pg} / \mathrm{ml}$; controls: $2734 \mathrm{pg} / \mathrm{ml}$, $366-13018 \mathrm{pg} / \mathrm{ml}, p<0.05)$ were significantly different.

3.4. Serum and Sputum Calprotectin Levels. Serum calprotectin concentrations (BO patients: $1416 \mathrm{ng} / \mathrm{ml}, 504-4161 \mathrm{ng} / \mathrm{ml}$; control subjects: $2342 \mathrm{ng} / \mathrm{ml}, 875-4331 \mathrm{ng} / \mathrm{ml}, p<0.05)$ were significantly lower in $\mathrm{BO}$ patients than in control subjects (Figure 3(a)).

In contrast, sputum calprotectin levels were significantly elevated in $\mathrm{BO}$ patients compared to control subjects (BO patients: $6429 \mathrm{ng} / \mathrm{ml}, 30.6-84000 \mathrm{ng} / \mathrm{ml}$; control subjects: $950 \mathrm{ng} / \mathrm{ml}, 135-2887 \mathrm{ng} / \mathrm{ml}, p<0.001)$ as shown in Figure 3(b).

There was a significant correlation of calprotectin with IL-8 levels (rho $=0.871 ; p<0.0001$ ) and of calprotectin with neutrophil counts, FEV1, MEF25, and the LCI (Table 2). There was no significant correlation of calprotectin with IL- $1 \beta$ and IL-6.
TABLE 1: Patient characteristics.

\begin{tabular}{lccc}
\hline & Controls & Patients with BO & $p$ value \\
\hline$n$ & 18 & 28 & - \\
Age (years) & $16.2(7-25)$ & $14.2(6-27)$ & n.s. \\
Sex (m/f) & $11 / 7$ & $16 / 12$ & n.s. \\
FVC (\%) & $93.0(71.6-105.8)$ & $80.7(25.5-107.4)$ & $<0.001$ \\
FEV1 (\%) & $97.9(83.3-114.9)$ & $61.9(14.6-105.2)$ & $<0.001$ \\
MEF25 (\%) & $103.1(44.9-137.1)$ & $26.7(6.7-107.9)$ & $<0.001$ \\
RV/TLC (\%) & $134.8(82.0-153.2)$ & $177.3(117.2-300.4)$ & $<0.001$ \\
LCI & $7.1(6.0-8.4)$ & $12.1(7.3-20.5)$ & $<0.001$ \\
\hline
\end{tabular}

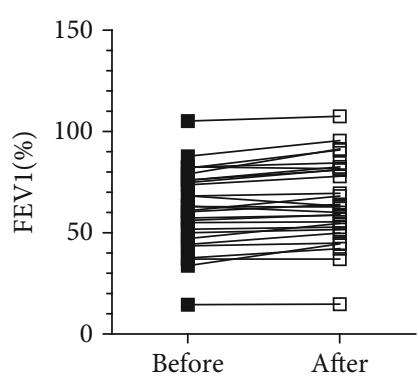

Figure 1: Jerkic et al.

\section{Discussion}

The term "bronchiolitis obliterans" describes common pathological alterations in the small airways following a variety of inciting diseases with different aetiologies and characteristics $[1,2]$. The process that leads to the observed pathology in the small airways is influenced not only by the initial insult but also by the localized inflammatory response and preexisting factors, such as nutritional status and genetic variants [2-4]. Previous studies have demonstrated an ongoing neutrophilic inflammation and increased proinflammatory markers, especially IL- 8 in the airways of patients with BO $[5,13,21]$. Nevertheless, there is an ongoing search for biomarkers that can be used to assess the risk and disease activity in a single patient. Calprotectin is a protein that is found extensively in the cytoplasm of neutrophils. It is released passively after cell death within the limits of an inflammatory process, and its value in inflammatory bowel disease is well established [14-16]. As expected, calprotectin was significantly increased in the sputum samples of the BO cohort compared to the control subjects. In addition, there was a significant correlation of calprotectin with neutrophil counts, IL- 8 levels, and the levels of the proinflammatory cytokines IL- $1 \beta$ and IL- 6 . These results suggest that calprotectin could be a reliable indicator of inflammatory processes within the small airways. The most significant correlation was found between IL- 8 and calprotectin. IL- 8 is produced by epithelial cells and has a chemotactic effect on neutrophils. The increase in both markers, IL-8 and neutrophils, strongly suggests persistent inflammation in the small airways in our BO cohort. Since our protocol excluded BO patients with acute respiratory infections and because some of them were already diagnosed with BO more than 10 years ago, it is very likely 


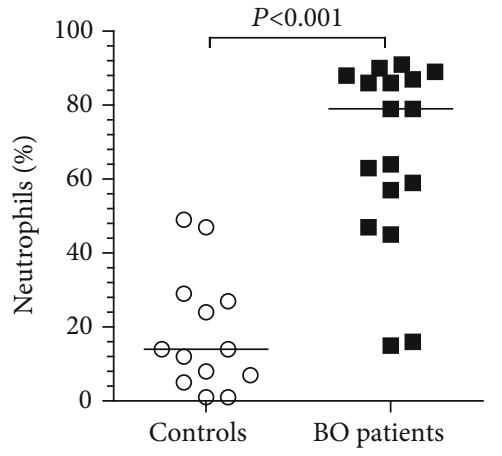

(a)

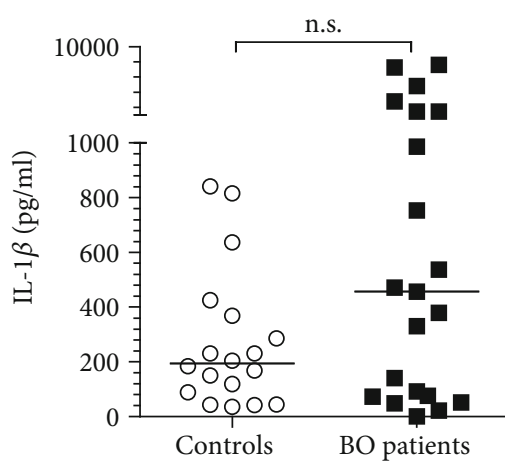

(c)

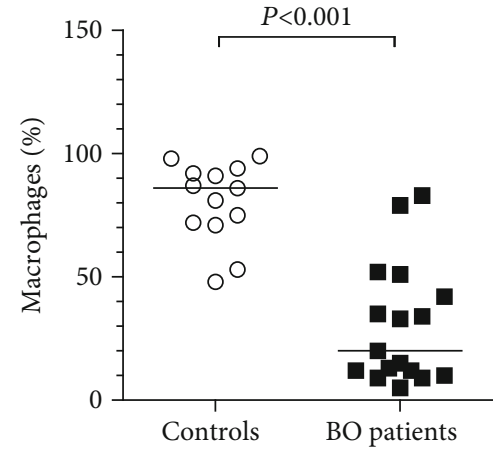

(b)

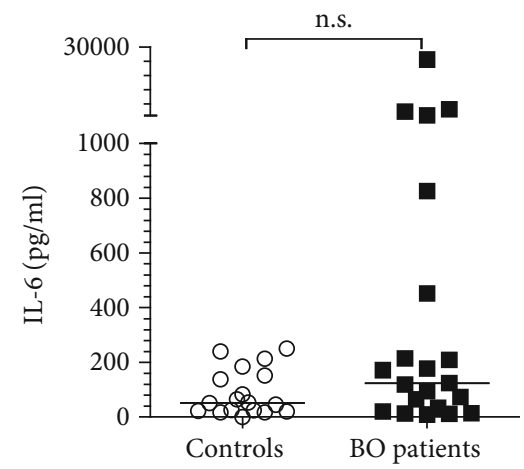

(d)

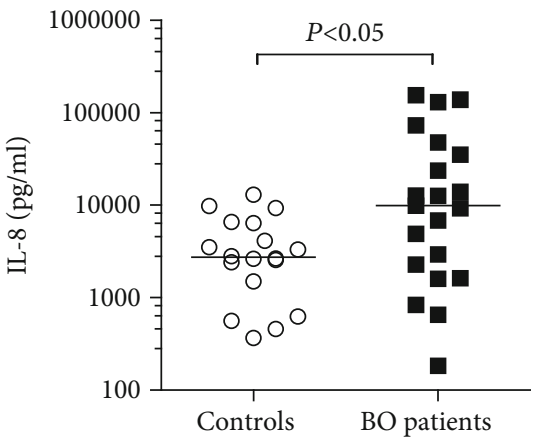

(e)

Figure 2: Jerkic et al.

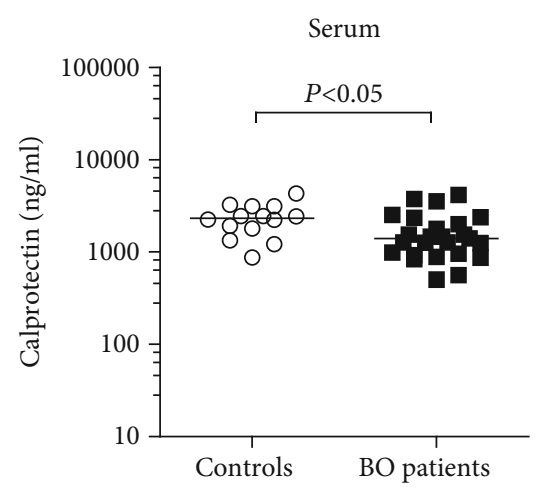

(a)

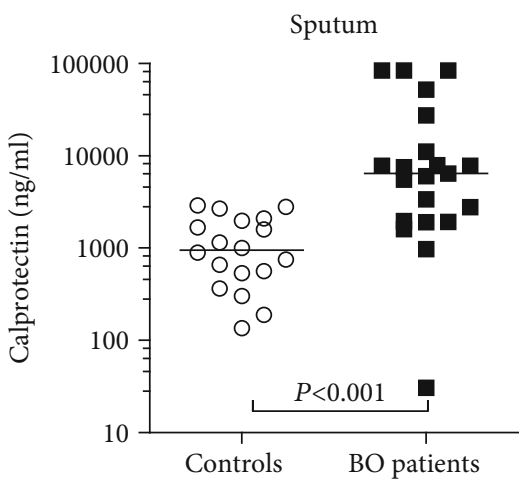

(b)

Figure 3: Jerkic et al. 
TABLE 2: Correlations with calprotectin.

\begin{tabular}{lc}
\hline & Correlation coefficient (rho) \\
\hline Neutrophils & $0.550^{* *}$ \\
IL-8 & $0.879^{* * *}$ \\
FVC & -0.173 \\
FEV1 & $-0.445^{* *}$ \\
MEF25 & $-0.445^{* *}$ \\
RV/TLC & 0.245 \\
LCI & $0.386^{*}$ \\
\hline
\end{tabular}

that there is an underlying chronic inflammatory process rather than inflammation triggered by an acute infection.

Michel et al. [22] investigated induced sputum in healthy control subjects before and 24 hours after lipopolysaccharide (LPS) inhalation and showed a correlation between increased neutrophil counts and calprotectin levels in the sputum. Repeated measurements showed reproducibly increased calprotectin levels; however, increased neutrophil counts were not correlated with the results of the first attempt [22]. This finding could indicate that there is an advantage in terms of the reproducibility of calprotectin levels compared to neutrophil counts in sputum. Studies by Gray et al. [23] suggested that calprotectin could indicate changes due to inflammatory processes. Calprotectin levels measured in the sputum of CF patients decreased significantly after antibiotic treatment for an acute exacerbation $[23,24]$. However, further investigations are necessary to reveal whether calprotectin levels measured in the sputum of $\mathrm{BO}$ patients decrease equally after antibiotic and anti-inflammatory treatments.

Further investigations in patients with CF have determined that increased serum calprotectin levels reflect inflammatory activity [25]. Presumably, increased serum calprotectin levels could occur in circulation either by leaky epithelial barriers at the site of inflammation or by increased recruitment of neutrophils from the bone marrow [24]. However, in our study, there was no increase in calprotectin levels in the serum samples of the $\mathrm{BO}$ cohort but a clear reduction in serum calprotectin levels. These results in $\mathrm{BO}$ patients may be because $\mathrm{BO}$ is associated with local inflammation that cannot be captured by systemic calprotectin levels; therefore, serum calprotectin is not a useful marker for airway inflammatory activity in BO. As an inflammatory marker, calprotectin has been well established in IBD $[15,16]$. Testing calprotectin levels is inexpensive, reproducible, and available. Therefore, calprotectin in the sputum could be used as an effective biomarker to identify patients with suspected diagnoses of BO. In addition, it would be helpful to investigate further whether there is an equally qualified method to measure sputum calprotectin levels that is not as time intensive as the present method.

Patients with BO typically develop small airway disease, which presents with significant flow limitations particularly affecting the small airways and typical signs of air trapping in distal areas of the lung [2-5]. Previous studies have revealed that there is a correlation between obstruction of the small airways and the neutrophil counts in the sputum and bronchoalveolar lavage (BAL) fluid. There is a clear rela- tionship between persistent neutrophilic inflammation and small airway disease in patients with $\mathrm{BO}, \mathrm{CF}$, and COPD $[5,21,26-28]$. There is evidence in CF that the LCI detects changes within the small airways much more sensitively than other tests [9]. The LCI in our BO cohort was significantly increased, and there was considerable inhomogeneity of ventilation, which is due to obstruction of the small airways. A significantly increased RV and RV/TLC and a decreased FEF 75 confirmed this finding. In addition, we found a significant correlation between the LCI and FEF 75, which suggests that both parameters characterize the extent of obstruction of the small airways. Previous studies have shown a continuous increase in the inhomogeneity of ventilation in patients with BOS measured by the LCI before any significant decline in spirometry was found $[11,12]$. Lahzami et al. [11] suggested that regular LCI measurements in patients after HSCT and LT could show the early stages of BOS; therefore, early treatment options could be established to prevent further fibrotic changes. However, further longitudinal measurements of the LCI are required to show whether the LCI is a sensitive and effective measure of the early stages of BO. In this study, there was a significant correlation between lung function test results and sputum calprotectin levels. A decreased FEV1 and FEF 75 and an increased LCI were clearly correlated with increased calprotectin levels in sputum samples from the $\mathrm{BO}$ cohort. BO patients with higher levels of calprotectin, indicating intensified inflammatory activity, showed worse obstruction in terms of lung function test results. However, there was no correlation between hyperinflation (RV and RV/TLC) and calprotectin levels.

\section{Conclusion}

BO is associated with chronic inflammation of the small airways that leads to obstructive airway disease. Due to persistent predominantly neutrophilic inflammation, calprotectin, which is released by neutrophils, is significantly increased in the sputum of patients with BO. Further investigations are required to confirm whether calprotectin is a sensible surrogate marker of neutrophilic inflammation to support the diagnosis of $\mathrm{BO}$ and to monitor the effects of antiinflammatory therapy in $\mathrm{BO}$.

\section{Data Availability}

All data of this paper are available on request. Please contact for this purpose Mrs Pera-Silvija Jerkic (Pera-Silvija.jerkic@kgu.de).

\section{Additional Points}

Key messages. What is the key question. Can calprotectin, which is a well-established and locally released marker, be used as sensitive indicator for persistent neutrophilic airway inflammation in patients with bronchiolitis obliterans? What is the bottom line. Calprotectin levels in sputum were significantly increased in patients with bronchiolitis obliterans (BO) and correlated strongly with IL-8 and an increased lung clearance index. Why read on. Elevated calprotectin levels 
reflect persistent neutrophilic airway inflammation, and calprotectin is a new and reliable biomarker in $\mathrm{BO}$.

\section{Disclosure}

This work was presented in part as a poster at the ERS in Paris. European Respiratory Journal 2018 52: Suppl. 62, PA4649.

\section{Conflicts of Interest}

The authors have the following conflicts to declare. Stefan Zielen received honoraria for lectures and consultations from the following companies: CSL Behring, AstraZeneca; SanofiAventis GmbH; Boehringer Ingelheim; Stallergenes Greer; Allergopharma GmbH \& Co. KG, Allergy Therapeutics, bene-Arzneimittel GmbH; Novartis AG; GlaxoSmithKline $\mathrm{GmbH}$; ALK-Abelló Arzneimittel GmbH; Lofarma GmbH, IMS Health $\mathrm{GmbH} \& \mathrm{Co}$; Engelhard Arzneimittel GmbH \& Co. KG and Biotest Pharma GmbH. Ralf Schubert received fees for consulting from Biotest Pharma $\mathrm{GmbH}$ and Vifor Pharma Deutschland GmbH. Martin Rosewich received fees for consulting from Allergy Therapeutics, Sanofi-Aventis $\mathrm{GmbH}$, GlaxoSmithKline GmbH, Engelhard Arzneimittel $\mathrm{GmbH} \&$ Co. KG, and Allergopharma GmbH \& Co. KG. There are no conflicts of interest to report for the authors Pera-Silvija Jerkic, Felix Michel., Helena Donath, and Eva Herrmann. The authors confirm that the above stated conflicts of interest did not cause any bias in the present publication.

\section{Acknowledgments}

The study was supported by a grant from the foundation "Stiftung Starke Lunge". This foundation is a nonprofit organization which supports scientific research on rare lung diseases (https://starkelunge.de). The authors acknowledge the assistance in sputum induction of Annalena Kochems.

\section{References}

[1] A. F. Barker, A. Bergeron, W. N. Rom, and M. I. Hertz, "Obliterative bronchiolitis," The New England Journal of Medicine, vol. 370, no. 19, pp. 1820-1828, 2014.

[2] G. Kurland and P. Michelson, "Bronchiolitis obliterans in children," Pediatric Pulmonology, vol. 39, no. 3, pp. 193-208, 2005.

[3] J. Yu, "Postinfectious bronchiolitis obliterans in children: lessons from bronchiolitis obliterans after lung transplantation and hematopoietic stem cell transplantation," Korean Journal of Pediatrics, vol. 58, no. 12, pp. 459-465, 2015.

[4] K. J. Smith and L. L. Fan, "Insights into post-infectious bronchiolitis obliterans in children," Thorax, vol. 61, no. 6, pp. 462-463, 2006.

[5] M. Rosewich, U. M. Zissler, T. Kheiri et al., "Airway inflammation in children and adolescents with bronchiolitis obliterans," Cytokine, vol. 73, no. 1, pp. 156-162, 2015.

[6] E. Kavaliunaite and P. Aurora, "Diagnosing and managing bronchiolitis obliterans in children," Expert Review of Respiratory Medicine, vol. 13, no. 5, pp. 481-488, 2019.
[7] P. Aurora, A. Bush, P. Gustafsson et al., "Multiple-breath washout as a marker of lung disease in preschool children with cystic fibrosis," American Journal of Respiratory and Critical Care Medicine, vol. 171, no. 3, pp. 249-256, 2005.

[8] A. Horsley, P. Gustafsson, K. A. Macleod et al., "Lung clearance index is a sensitive, repeatable and practical measure of airways disease in adults with cystic fibrosis," Thorax, vol. 63, no. 2, pp. 135-140, 2008.

[9] L. Kent, P. Reix, J. A. Innes et al., "Lung clearance index: evidence for use in clinical trials in cystic fibrosis," Journal of Cystic Fibrosis, vol. 13, no. 2, pp. 123-138, 2014.

[10] J. H. Rayment, S. Stanojevic, S. D. Davis, G. Retsch-Bogart, and F. Ratjen, "Lung clearance index to monitor treatment response in pulmonary exacerbations in preschool children with cystic fibrosis," Thorax, vol. 73, no. 5, pp. 451-458, 2018.

[11] S. Lahzami, R. E. Schoeffel, V. Pechey et al., "Small airways function declines after allogeneic haematopoietic stem cell transplantation," The European Respiratory Journal, vol. 38, no. 5, pp. 1180-1188, 2011.

[12] S. Nyilas, L. Baumeler, M. Tamm et al., "Inert gas washout in bronchiolitis obliterans following hematopoietic cell transplantation," Chest, vol. 154, no. 1, pp. 157-168, 2018.

[13] Y. Y. Koh, D. E. Jung, J. Y. Koh, J. Y. Kim, Y. Yoo, and C. K. Kim, "Bronchoalveolar cellularity and interleukin-8 levels in measles bronchiolitis obliterans," Chest, vol. 131, no. 5, pp. 1454-1460, 2007.

[14] A. Voganatsi, A. Panyutich, K. T. Miyasaki, and R. K. Murthy, "Mechanism of extracellular release of human neutrophil calprotectin complex," Journal of Leukocyte Biology, vol. 70, no. 1, pp. 130-134, 2001.

[15] B. E. Sands, "Biomarkers of inflammation in inflammatory bowel disease," Gastroenterology, vol. 149, no. 5, pp. 12751285.e2, 2015.

[16] G. Rosenfeld, A. J. Greenup, A. Round et al., "FOCUS: Future of fecal calprotectin utility study in inflammatory bowel disease," World Journal of Gastroenterology, vol. 22, no. 36, pp. 8211-8218, 2016.

[17] J. Wanger, J. L. Clausen, A. Coates et al., "Standardisation of the measurement of lung volumes," The European Respiratory Journal, vol. 26, no. 3, pp. 511-522, 2005.

[18] J. Schulze, S. Voss, U. Zissler, M. A. Rose, S. Zielen, and R. Schubert, "Airway responses and inflammation in subjects with asthma after four days of repeated high-single-dose allergen challenge," Respiratory Research, vol. 13, no. 1, p. 78, 2012.

[19] S. Koc-Günel, R. Schubert, S. Zielen, and M. Rosewich, "Cell distribution and cytokine levels in induced sputum from healthy subjects and patients with asthma after using different nebulizer techniques," BMC Pulmonary Medicine, vol. 18, no. 1, p. 115, 2018.

[20] O. Eickmeier, M. Huebner, E. Herrmann et al., "Sputum biomarker profiles in cystic fibrosis (CF) and chronic obstructive pulmonary disease (COPD) and association between pulmonary function," Cytokine, vol. 50, no. 2, pp. 152-157, 2010.

[21] J. Eckrich, E. Herrmann, S. Voss, R. Schubert, S. Zielen, and M. Rosewich, "Short-term variation of lung function and airway inflammation in children and adolescents with bronchiolitis obliterans," Lung, vol. 194, no. 4, pp. 571-579, 2016.

[22] O. Michel, V. Doyen, B. Leroy et al., "Expression of calgranulin A/B heterodimer after acute inhalation of endotoxin: proteomic approach and validation," BMC Pulmonary Medicine, vol. 13 , no. 1 , p. $65,2013$. 
[23] R. D. Gray, M. Imrie, A. C. Boyd, D. Porteous, J. A. Innes, and A. P. Greening, "Sputum and serum calprotectin are useful biomarkers during CF exacerbation," Journal of Cystic Fibrosis, vol. 9, no. 3, pp. 193-198, 2010.

[24] Z. Schnapp, C. Hartman, G. Livnat, M. Shteinberg, and Y. Elenberg, "Decreased fecal calprotectin levels in cystic fibrosis patients after antibiotic treatment for respiratory exacerbation," Journal of Pediatric Gastroenterology and Nutrition, vol. 68, no. 2, pp. 282-284, 2019.

[25] M. Pruenster, T. Vogl, J. Roth, and M. Sperandio, "S100A8/A9: from basic science to clinical application," Pharmacology \& Therapeutics, vol. 167, pp. 120-131, 2016.

[26] S. L. Peterson-Carmichael, W. T. Harris, R. Goel et al., "Association of lower airway inflammation with physiologic findings in young children with cystic fibrosis," Pediatric Pulmonology, vol. 44, no. 5, pp. 503-511, 2009.

[27] J. Eckrich, U. M. Zissler, F. Serve et al., "Airway inflammation in mild cystic fibrosis," Journal of Cystic Fibrosis, vol. 16, no. 1, pp. 107-115, 2017.

[28] R. A. O'Donnell, C. Peebles, J. A. Ward et al., "Relationship between peripheral airway dysfunction, airway obstruction, and neutrophilic inflammation in COPD," Thorax, vol. 59, no. 10 , pp. $837-842,2004$. 